\title{
Possible local treatment for liver metastases of adenoid cystic carcinoma (ACC): single-centre experience and literature review
}

\author{
Yiling Zheng ${ }^{1}$, Yuqin $\mathrm{He}^{1}$, Fan $\mathrm{Wu}^{1}$, Mei Liu ${ }^{2}$, Liming Wang ${ }^{1}$, Jianxiong $\mathrm{Wu}^{1}$ \\ ${ }^{1}$ Department of Hepatobiliary Surgery of Cancer Hospital, ${ }^{2}$ Laboratory of Cell and Molecular Biology and State Key Laboratory of Molecular \\ Oncology, National Cancer Center/National Clinical Research Center for Cancer/Cancer Hospital, Chinese Academy of Medical Sciences and \\ Peking Union Medical College, Beijing, China \\ Contributions: (I) Conception and design: L Wang, J Wu; (II) Administrative support: F Wu; (III) Provision of study material of patients: L Wang, \\ F Wu; (IV) Collection and assembly of data: Y Zheng, Y He; (V) Data analysis and interpretation: Y Zheng, Y He; (VI) Manuscript writing: All \\ authors; (VII) Final approval of manuscript: All authors. \\ Correspondence to: Liming Wang, MD, PhD; Jianxiong Wu, MD, PhD. Department of Hepatobiliary Surgery of Cancer Hospital, No.17 Panjiayuan \\ Nanli, Chaoyang District, Beijing, China. Email: stewen_wang@sina.com; dr_wujx@126.com.
}

\begin{abstract}
Background: Adenoid cystic carcinoma (ACC) is a relatively indolent cancer, for which the major cause of death is distant metastases. The median survival time of patients with distant metastases of ACC is approximately 20 months. Although the liver is not the most common site of secondary ACC, effective treatment of liver metastases could improve patient survival. This study explored the clinical features and treatment of liver metastases of ACC in one centre and performed a literature review.

Methods: This is a retrospective cohort study. The records of 25 ACC patients with liver metastases in our hospital from January 2000 to September 2018 and 13 case reports (from 2000 to 2018) from PubMed published in English were reviewed together. Survival curves were estimated by the Kaplan-Meier method. Multivariate cox regression analysis was used to identify risk factors for overall survival (OS).

Results: Patients with liver metastases of ACC had a poor prognosis. The median survival time of patients with ACC liver metastases was 14 months (4-26 months), and the 1-, 2-, and 3-year survival rates were $55.8 \%, 28.5 \%$, and $15.2 \%$, respectively. Isolated liver metastases were more likely to benefit from local treatment, such as surgery and radiofrequency ablation (RFA), than multifocal metastases. The prognosis was worse for patients who received systemic treatment for multifocal liver metastases than for those who received other treatment. Synchronous liver metastases were the predictive factor for OS both in univariate and multivariate analysis.
\end{abstract}

Conclusions: Local treatment, such as surgical resection or RFA, may prolong the survival time of ACC patients with liver metastases. The definition of resectability for liver metastases needs further investigation.

Keywords: Adenoid cystic carcinoma (ACC); distant metastases; liver metastases

Submitted Feb 14, 2020. Accepted for publication Jun 28, 2020.

doi: $10.21037 /$ tcr-20-1028

View this article at: http://dx.doi.org/10.21037/tcr-20-1028

\section{Introduction}

Adenoid cystic carcinoma (ACC) is a rare type of cancer that most often occurs in the salivary glands, breast, trachea, lacrimal gland, paranasal sinuses and Bartholin gland. ACC is composed of glandular epithelial cells and myoepithelial cells with tubular, glandular and solid structures. The two types of cells in ACC are hyperchromatic cells and those that have angular nuclei, and these cells frequently have clear cytoplasm (1). Though a low-grade malignancy, ACC has malignant characteristics such as vessel infiltration, perineural infiltration, local recurrence and distant metastases. Primary ACC is often managed with surgery, and some medical centres prefer postoperative radiotherapy 
to reduce the incidence of local recurrence (2), given that the local recurrence rate is approximately $40 \%$ at 5 years. T4 stage, nodal involvement, solid histology, perineural invasion (PNI) and positive surgical margin are considered to be negative prognostic factors (3). Distant metastasis (DM) eventually occurs in as many as $40 \%$ of patients (3); with a 36-months follow-up, distant metastases developed in $25(30.5 \%)$ of 82 patients (4). Despite the primary lowgrade malignant nature of primary ACC, the survival rate of ACC is rather low, with a 10 -year survival rate of less than $50 \%$, which is due to distant metastases (5). Therefore, it is important to prevent metastases and improve the effectiveness of treatment for metastases of ACC.

However, the high rate of distant metastases of ACC does not seem be associated with local recurrence (6). The most common sites of metastases are the lung and bone, followed by the brain and liver. Lung metastases occur in $70 \%$ of patients with distant metastases (7). The liver is not the most common metastatic site of ACC, but there is no standardized treatment for liver metastases, resulting in a poor prognosis (8). Most of the literature is composed of case reports without systematic studies; although the National Comprehensive Cancer Network (NCCN) has no relevant treatment guidelines, locoregional therapy such as radiofrequency ablation (RFA) is commonly used in secondary liver metastasis (9) including liver metastasis of ACC. Therefore, we reviewed the cases of ACC with liver metastases in our centre along with the literature, with the aim of providing some clues for the effective treatment of ACC with liver metastases in the future. We present the following article in accordance with the STROBE reporting checklist (available at http://dx.doi.org/10.21037/tcr-201028).

\section{Methods}

\section{Patients}

This is a retrospective observational cohort study that was performed in the Cancer Hospital, Chinese Academy of Medical Sciences and Peking Union Medical College. A total of 835 patients were diagnosed with ACC from January 2000 to September 2018; among them, 194 patients had distant metastases, and 26 patients were identified as having liver metastases of ACC, which was confirmed by radiographic findings consistent with metastatic disease or biopsy. Among these patients, one patient was excluded from our study because he died from prostate cancer. Informed consent was signed by the patients before treatment. The research was in compliance of the Declaration of Helsinki (as revised in 2013). Patients' data was retrieved from hospital medical record system and the patient's personal data have been secured. Ethical approval was obtained from the Institutional Review Board of the Cancer Hospital, Chinese Academy of Medical Sciences (ID: NCC2019C-027).

\section{Follow up}

Clinical and demographic information, including clinical manifestations, pathological characteristics, treatment options, and follow-up information, was obtained through hospital records or by direct contact with patients or family members. Patients were checked for progressive disease by diagnostic imaging, such as computed tomography (CT) and magnetic resonance imaging (MRI) every 6 weeks after each treatment for liver metastasis of ACC and every 3 months for 2 years after stable disease, then every 6 months afterwards. The observation period was from the date of first treatment until the date of death or censoring. Progression-free survival (PFS) and overall survival (OS) was both measured from the date of the initial treatment of liver metastatic tumour. Disease progression was defined as the enlargement of initial liver metastatic tumour or the occurrence of new DM after the first treatment of liver metastasis of ACC. PFS was censored at the date of diagnosis of progression or last follow-up, and OS was censored at the date of death or last follow-up.

We divided all treatments into local treatment, transcatheter arterial chemoembolization (TACE) and systematic treatment groups. Local treatment included surgery and RFA, while systematic treatment included chemotherapy and biotherapy. The Response Evaluation Criteria in Solid Tumour version 1.1 (RECIST 1.1) (10) was applied to assess the change in tumour burden 6 weeks after each non-surgical treatment for liver metastases.

\section{Statistical analysis}

Statistical analysis was performed using IBM SPSS 25.0 (IBM, Armonk, New York, USA). Fisher's exact test or Chi square test were used for comparisons of categorical variable distributions among liver metastasis patterns and treatment options. To gain a better understanding of the clinical features of and impact of treatment options on liver metastases, another 13 case reports (from 2000 
Table 1 Clinical characters of adenoid cystic carcinoma (ACC) of liver metastases

\begin{tabular}{|c|c|}
\hline Character & Outcome \\
\hline Age (years) & $55.92 \pm 8.71$ \\
\hline Female:male & $14: 11$ \\
\hline \multicolumn{2}{|l|}{ Original site } \\
\hline Nasopharynx & $6 / 25(24.0 \%)$ \\
\hline Submandibular & $6 / 25(24.0 \%)$ \\
\hline Trachea & $2 / 25(8.0 \%)$ \\
\hline Lung & $3 / 25(12.0 \%)$ \\
\hline Larynx & $2 / 25$ (8.0\%) \\
\hline Lacrimal gland & $1 / 25(4.0 \%)$ \\
\hline Parotid gland & $1 / 25(4.0 \%)$ \\
\hline Breast & $1 / 25(4.0 \%)$ \\
\hline Tongue & $1 / 25(4.0 \%)$ \\
\hline Floor of mouth & $1 / 25(4.0 \%)$ \\
\hline Orbital & $1 / 25(4.0 \%)$ \\
\hline \multicolumn{2}{|l|}{ Primary ACC treatment } \\
\hline Surgery & $7 / 25(28.0 \%)$ \\
\hline Radiotherapy & $2 / 25(8.0 \%)$ \\
\hline Surgery + radiotherapy & $10 / 25(40.0 \%)$ \\
\hline Surgery + chemotherapy & $2 / 25$ (8.0\%) \\
\hline Chemotherapy & $4 / 25(16.0 \%)$ \\
\hline \multicolumn{2}{|l|}{ Recurrence of original site } \\
\hline Yes & $8 / 25(32.0 \%)$ \\
\hline No & $17 / 25(68.0 \%)$ \\
\hline \multicolumn{2}{|l|}{ Liver metastases } \\
\hline Isolated liver metastases & $6 / 25(24.0 \%)$ \\
\hline Multifocal liver metastases & $19 / 25(76.0 \%)$ \\
\hline Synchronous liver metastases & $5 / 25(20.0 \%)$ \\
\hline Metachronous liver metastases & $20 / 25(80.0 \%)$ \\
\hline
\end{tabular}

to 2018) from PubMed independently searched by two researchers were reviewed together with our cases. A retrospective survival analysis was performed with KaplanMeier survival curves. Differences between the survival curves were examined by log-rank tests. The factors of OS used Cox's proportional hazards model as multivariate analysis and stepwise analysis. The accepted level of significance was $\mathrm{P}<0.05$.

\section{Results \\ Clinical analysis of primary ACC}

Of the 25 patients in our study, 13 were male, and 12 were female. The average age was 55.92 \pm 8.71 (IQR: 44-67) years. The most common original sites were the submandibular gland (6 patients) and nasopharynx (6 patients); the other sites are shown in Table 1. The patients were followed up until July 2019, and the median follow-up duration was 32 (IQR: 14-66) months.

For primary ACC, 4 patients who underwent R1 surgery received radiotherapy after surgery, only one had recurrence in the primary site and underwent surgery again. Four patients underwent chemotherapy with synchronous metastases, which was defined as the original ACC found with metastases in other organs at the same time. Another patient with synchronous metastases underwent surgery for primary lung ACC and TACE for liver metastases, and this patient developed recurrence 5 months later and underwent chemotherapy and radiotherapy instead. All 5 synchronous metastasis patients had progressive disease during the follow-up. Two patients received radiotherapy rather than undergoing surgery for primary ACC, one of whom developed recurrence after 12 months and underwent radiotherapy again.

Liver metastases were confirmed by radiographic findings or biopsy and were described as equal or hypoechoic nodules by B-ultrasound, irregular low-density nodules by computerized tomography (CT), or nodules with heterogeneous signal by magnetic resonance imaging (MRI). A dynamic MRI of liver metastases is shown in Figure 1. A pathologic diagnosis of adenoid cystic liver metastases is shown in Figure 2. The median time until a diagnosis of liver metastasis was 18 (IQR: 3-44.5) months from the first diagnosis ACC.

\section{Clinical characteristics of liver metastases}

The clinical characteristics of liver metastases of ACC are shown in Table 1.

At the final follow-up date, 4 patients were still alive, and 2 patients were lost to follow-up and censored. A total of $73.08 \%$ of the patients had multifocal liver metastases. Five patients (19.23\%) had synchronous liver metastases, and among them, 3 patients had lung metastases at the 

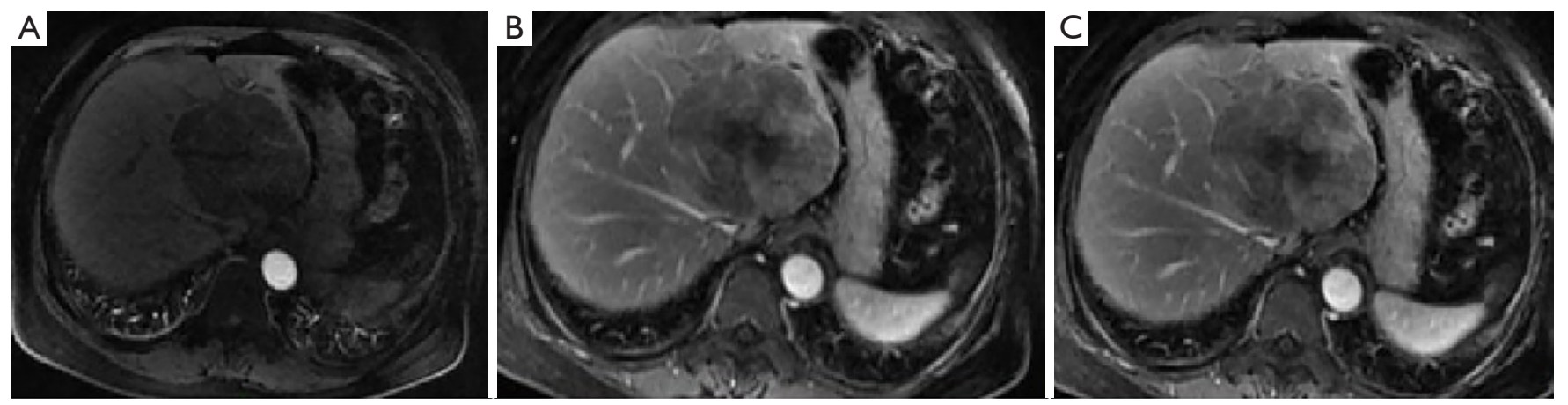

Figure 1 Dynamic magnetic resonance imaging of the liver. (A) At the arterial phase: showed a well-delimited, slightly enhanced lesion in left liver. (B) At the venous phase: showed progressive enhancement of the lesion. (C) At the 3-minutes delayed venous phase: lesion appeared slightly less enhancing compared with the surrounding liver parenchyma with no enhancement in the center.
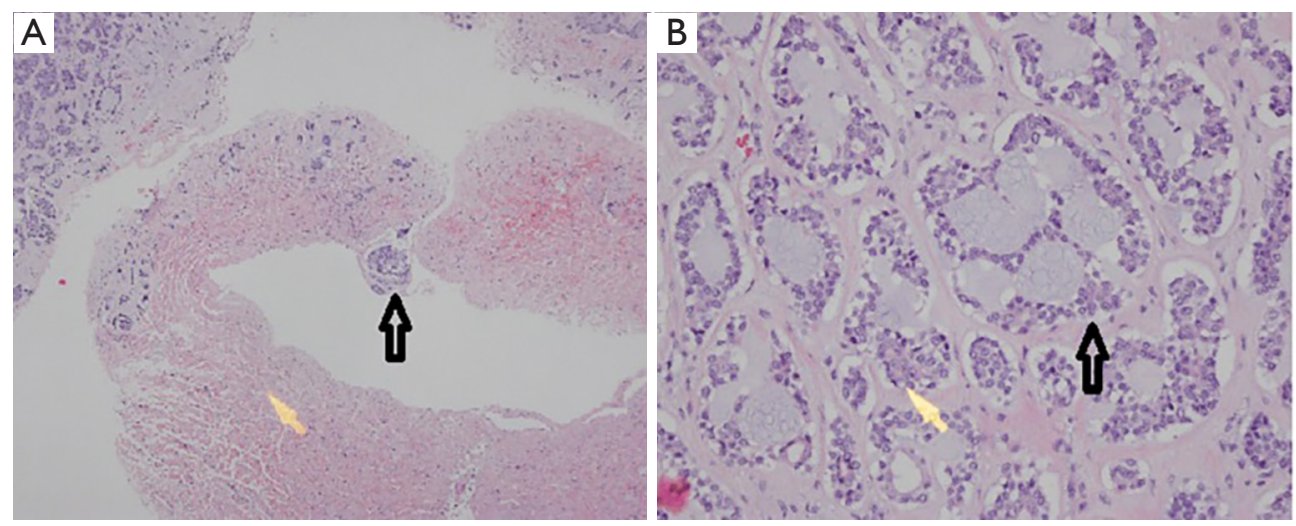

Figure $2 \mathrm{H} \& \mathrm{E}$ staining at $4 \times(\mathrm{A})$ and $10 \times$ (B) magnification. The left image (A) shows tumor infiltration of the vessel (black arrow). The right image (B) shows glandular structures with mucin in the lumen (black arrow) characteristic of adenoid cystic carcinoma. The yellow arrows are microscopic markers, independent of tumor characteristics.

same time. Ten patients were found to have lung metastases before liver metastases.

The other 13 case reports in PubMed were reviewed in Table 2 (5,8,11-21).

\section{Treatment for liver metastases}

The treatments for liver metastases in our centre are listed in Tables 3,4. One patient declined treatment after being diagnosed with liver metastases. The response of nonsurgical treatment was judged by RECIST 1.1.

Only 2 patients in our centre underwent local treatment without any systematic therapy. One patient with multifocal liver metastases who underwent RFA three times maintained stable disease, and another patient who underwent surgery with isolated metastases achieved no recurrence and no other new metastasis. In the reviewed literature, 4 patients underwent surgery for liver metastases. One had isolated liver metastases, one had isolated liver metastases with lung metastases, and the other two had multifocal metastases. All of the patients did not have progressive disease in the first 6 weeks of evaluation according to the literature.

In our centre, 8 patients received TACE alone without any benefits, in other words, all of them had progressive disease. The other one patient who combined TACE with chemotherapy also did not benefit from the treatment. In the reviewed literature, one patient remained stable 10 months after combined TACE and chemotherapy.

In our centre, 9 patients underwent chemotherapy alone and had progressive disease. Four patients received combined local treatment and chemotherapy; among them, two patients with isolated liver metastases had stable disease. 


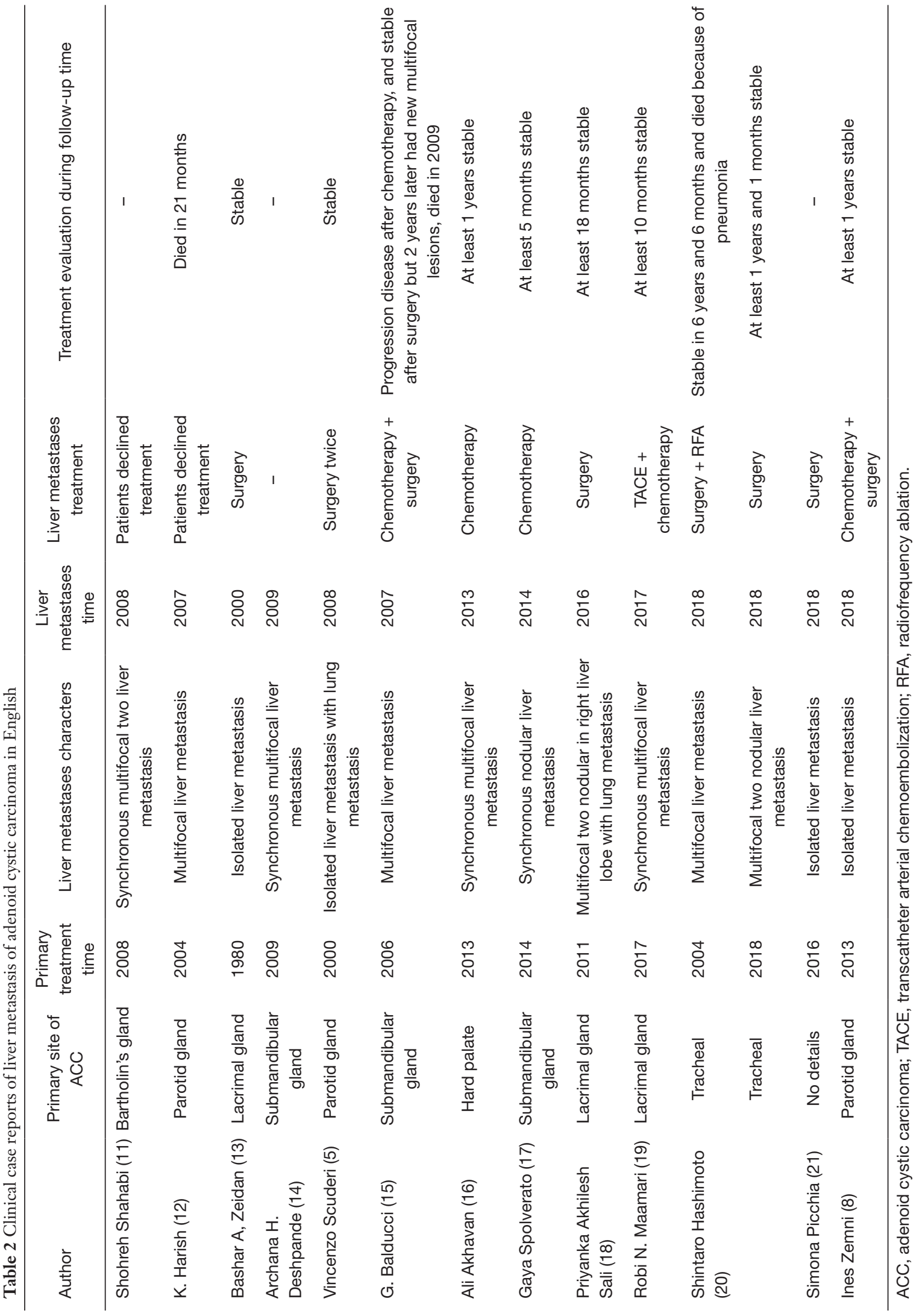


Table 3 Clinical treatment and evaluation

\begin{tabular}{lcl}
\hline Treatment & Number of patients & Treatment evaluation \\
\hline Isolated liver metastases & $1 / 25$ & Progression disease after chemotherapy, complete response now after RFA \\
Surgery + chemotherapy + RFA & $1 / 25$ & Progression disease \\
Chemotherapy + TACE & $1 / 25$ & Stable disease \\
RFA + chemotherapy & $1 / 25$ & Progression disease \\
Chemotherapy & $1 / 25$ & Progression disease \\
TACE & $1 / 25$ & No recurrence and no other new metastasis \\
Surgery & & \\
Multifocal liver metastases & $7 / 25$ & Progression disease \\
TACE & $8 / 25$ & Progression disease \\
Chemotherapy & $1 / 25$ & Stable disease \\
RFA & $1 / 25$ & Progression disease \\
Chemotherapy + RFA & $1 / 25$ & Progression disease until biotherapy, stable disease now \\
Surgery + TACE + RFA + & & \\
chemotherapy + biotherapy &
\end{tabular}

TACE, transcatheter arterial chemoembolization; RFA, radiofrequency ablation.

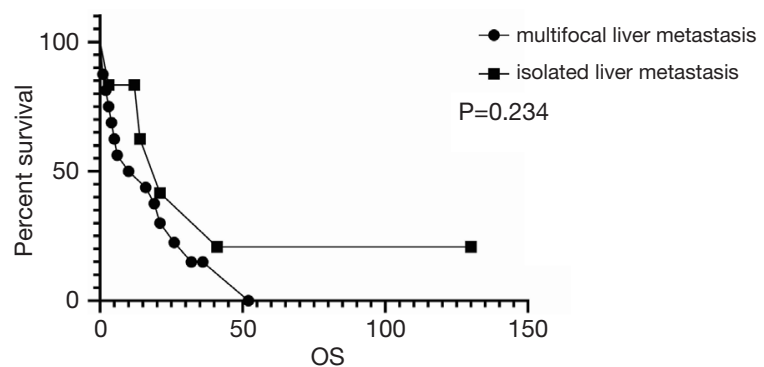

Figure 3 Kaplan-Meier survival curves for multifocal and isolated liver metastases.

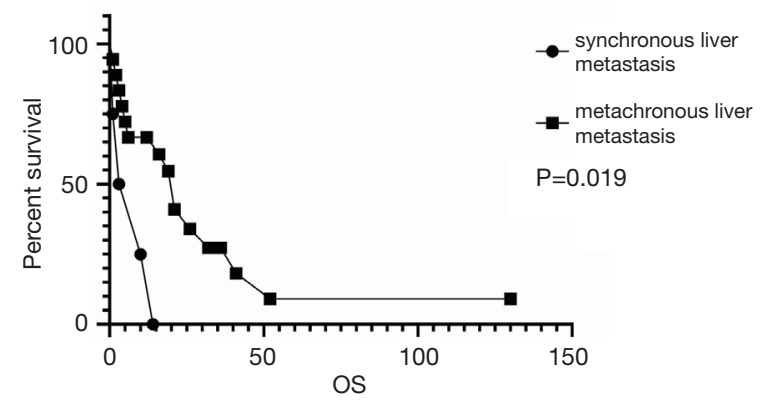

Figure 4 Kaplan-Meier survival curves for synchronous liver metastases and metachronous liver metastases.
One patient had a new hepatic lesion 7 years after radical liver metastasis surgery, then she received chemotherapy but still had poor response, after multidisciplinary discussion she underwent RFA twice and had stable disease until now. Another patient had RFA first and took chemotherapy as adjuvant therapy after liver metastasis, which led to stable disease now. Two patients with multifocal metastases did not respond to chemotherapy. Whether local treatment was performed before or after chemotherapy, both two patients had poor response to chemotherapy or local treatment.

As shown in Tables 2 and 3, different patterns of liver metastases should receive different treatments. For resectable lesions, local treatment has shown benefits without short-term recurrence or metastasis at another site. In particular, for multifocal liver metastases, TACE rarely showed any significant benefits.

\section{Survival of patients with liver metastases}

As shown in Figure 3, the survival analysis showed no difference between multifocal and isolated liver metastases. However, synchronous liver metastases had worse survival than metachronous liver metastases, as shown in Figure 4. 
Treatment response was dichotomized into stable (including complete response, partial response, stable disease and radical surgical resection without recurrence

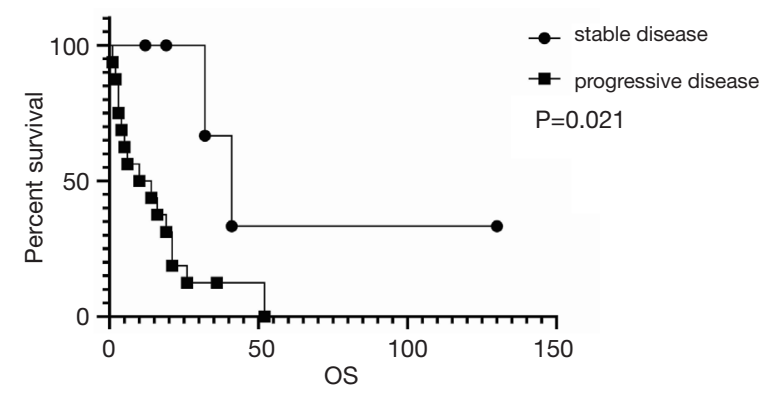

Figure 5 Kaplan-Meier survival curves for stable disease and progressive disease.

Table 4 Treatment and treatment evaluation in summarizes

\begin{tabular}{|c|c|c|}
\hline \multirow[b]{2}{*}{ Treatment } & \multicolumn{2}{|c|}{ Treatment evaluation } \\
\hline & $\begin{array}{l}\text { Stable (number } \\
\text { of patients) }\end{array}$ & $\begin{array}{c}\text { Progressive } \\
\text { (number of patients) }\end{array}$ \\
\hline \multicolumn{3}{|l|}{ Isolated liver metastases } \\
\hline Local treatment* & 1 & 0 \\
\hline TACE & 0 & 7 \\
\hline Systematic treatment & 1 & 9 \\
\hline \multicolumn{3}{|c|}{ Multifocal liver metastases } \\
\hline Local treatment* & 1 & 0 \\
\hline TACE & 0 & 1 \\
\hline Systematic treatment & 2 & 2 \\
\hline
\end{tabular}

or new DM) and progressive disease. As shown in Figure 5, response to treatment was associated with survival, and effective treatment led to a better prognosis. Patients with progressive disease had a median PFS of less than 6 weeks, while the median PFS for stable patients was 32 months. The median OS of ACC liver metastases in our centre was 14 (IQR: 4-26) months, and the 1-, 2-, and 3-year OS rates were $55.8 \%, 28.5 \%$, and $15.2 \%$, respectively.

In other studies (Table 4), local treatment seemed to be more beneficial for patients than other treatments $(\mathrm{P}=0.008)$. Age, gender, patterns of liver metastases, number of liver metastases and treatment evaluation were included in univariate and multivariate cox regression analysis about OS (Table 5). Synchronous liver metastases were the predictive factor for OS both in univariate (HR: 4.292, 95\% CI: 1.201-15.339, $\mathrm{P}=0.025)$ and multivariate analysis ( $\mathrm{HR}$ : 4.013, 95\% CI: $1.060-15.199, \mathrm{P}=0.041$ ).

\section{Discussion}

Our study is the first cohort study to demonstrate that surgery and RFA might be beneficial for patients with liver metastasis of ACC while TACE seems to be not an effective treatment for liver metastasis of ACC. Consistent with the results of literature reviewed in PubMed, the results of our study might be applied to other medical centres as well.

ACC is a relatively indolent cancer, which means that its 5-year survival rate is high relative to other malignant tumours. Primary ACC is often found in the salivary gland, and the main treatments are surgery and radiotherapy. Local recurrence occurs in $26 \%$ of all ACC patients (22). Positive margins are the only statistically significant parameter that leads to local recurrence.

However, distant metastases are common and lead to have poor long-term survival rate. Distant metastases can occur despite obtaining primary tumour control and were

Table 5 Multivariate cox regression analysis for factors predicting overall survival

\begin{tabular}{|c|c|c|c|c|}
\hline Factor & \multicolumn{2}{|c|}{ Univariate analysis } & \multicolumn{2}{|c|}{ Multivariate analysis } \\
\hline Age & $0.977(0.927-1.031)$ & 0.397 & & \\
\hline Female & $0.495(0.176-1.394)$ & 0.183 & & \\
\hline Synchronous liver metastases & 4.292 (1.201-15.339) & $0.025^{\star}$ & 4.013 (1.060-15.199) & $0.041^{*}$ \\
\hline Progressive disease & 4.816 (1.069-21.696) & $0.041^{*}$ & $4.178(0.901-19.376)$ & 0.068 \\
\hline
\end{tabular}

*Significance at $\mathrm{P}<0.05$. 
identified in $45 \%$ of patients during the follow-up period at a mean of $100 \pm 79$ months after the initial therapy (20), even in patients without any evidence of locoregional treatment failure. In a cohort study of 160 ACC patients, the disease-specific survival rates at 5,10, and 15 years was $89 \%, 67.4 \%$, and $39.6 \%$ (23), respectively. As the most important factor influencing survival, DM has a statistically significant impact on final patient outcome (22). One study showed that the DM rate was not affected by primary site, $\mathrm{T}$ stage, histologic growth pattern, or local recurrence (6), but a positive surgical margin was significantly associated with DM. For ACC of the salivary glands, DM occurs most often in the lung, followed by other organs. The average time between the occurrence of metastases in the lung and death was 32.3 months, and the time between the occurrence of metastases elsewhere and death was 20.6 months (24). The liver is not a common organ of metastasis for ACC compared with the lung; there are only a few case reports (Table 2) of liver metastases or coincidental lung and liver metastases; furthermore, one study showed that lung metastases account for $94.4 \%$ of all DMs (7).

As DM seems to be the most important factor for OS, effective prevention and treatment for distant metastases is essential. At the initial treatment, a negative surgical margin should be obtained. A few studies have shown that radiotherapy might be effective in achieving local control $(2,25)$, yet chemotherapy is ineffective for the management of secondary ACC in most cases (16).

Although patients with distant metastases are in the terminal stage, effective treatment for liver metastases improved the survival outcomes in our study. Furthermore, patients who received local treatment, such as surgery and RFA, had survival benefits; isolated liver metastases treated with liver resection especially improved the survival rate. However, if liver metastases were accompanied by metastases at other sites, additional factors need to be considered. The definition of resectability might not be limited to isolated liver metastases because multiple liver metastases can also be surgically treated (15), even recurrent liver metastases (5). The extent of resection and the function of the remnant liver should be considered, and a multidisciplinary team should be involved in evaluating the treatment options. Expanding the definition and criteria for resectability for patients with liver metastases of ACC might lead to improved clinical outcomes compared to treatment with other adjunctive and adjuvant approaches.

RFA is widely applicable for secondary liver metastasis, especially for multiple colorectal liver metastasis. In our study, among the five patients who underwent RFA, four patients had stable disease. RFA seems to be an alternative method for treating liver metastasis of ACC. One study believed that RFA should obtain a margin size of at least $5 \mathrm{~mm}$ and be used for only solitary and small (less than $3 \mathrm{~cm}$ ) hepatic metastases (9). However, the margins of RFA in our centre were not well defined; in the future, the tumour size, number of tumours and width of the margin should be carefully recorded to make further conclusions.

There is no evidence that liver metastasis patients would benefit from TACE, in both our centre and in the literature. All patients in our centre who received TACE had disease progression. On the other hand, patients who received systematic treatment had a short median survival time of approximately 9 months, and approximately $90 \%$ of these patients died during the follow-up; in contrast, the patients who received local treatment were still alive. TACE is a common therapy for hepatic malignant tumour, especially for multiple hepatocellular carcinomas. Hepatic tumour with abundant arterial blood supply responses well to TACE. In the liver metastasis of ACC, the character of intra-tumour hypo-vascular might lead to poor response to TACE. One study indicated that the slow growth of kinetics of ACC resulted in low response rates to cytotoxic chemotherapy for metastatic disease (26). One study (27) showed that SIM (simvastatin) with an miR-21 inhibitor may be beneficial for distant metastases of ACC and might be a new strategy for the clinical treatment of ACC. Another study (28) showed that bortezomib (an inhibitor of the $26 \mathrm{~S}$ proteasome and $\mathrm{NF}-\mathrm{kB}$ ) resulted in disease stabilization in a high percentage of ACC patients with DM. To date, there are few studies about applying chemotherapy to treat distant metastases of ACC, and more research is needed on effective treatments for distant metastases of ACC.

There are three limitations in our study. The first is the small sample size. Although our study has the largest sample size to date of the studies in the literature, our sample size could still not obtain significant differences when comparing different treatments. Further studies will need to include more cases to expand the scope of the feasibility and practicability of treatment options. Second, because of its retrospective nature and lack of official guidelines, we could not avoid bias from the doctors' preference for treatment. However, it is difficult to implement a prospective study since liver metastasis of ACC is so rare. Finally, ACC has three kinds of basic growth patterns: tubular, cribriform, and solid. The solid type correlates with the most aggressive 
ACC, although these patterns are often mixed. We did not have these data in our study, so we could not analyse whether the basic growth pattern affected liver metastasis of ACC.

\section{Conclusions}

Our study provided some clues that local treatment might be effective for liver metastases of ACC, but more cases and studies are needed to expand the scope of the feasibility and practicability of this treatment option.

\section{Acknowledgments}

We gratefully acknowledge Qing Fang for his help with the pathological studies in this study.

Funding: None.

\section{Footnote}

Reporting Checklist: The authors have completed the STROBE reporting checklist. Available at http://dx.doi. org/10.21037/tcr-20-1028

Data Sharing Statement: Available at http://dx.doi. org/10.21037/tcr-20-1028

Conflicts of Interest: All authors have completed the ICMJE uniform disclosure form (available at http://dx.doi. org/10.21037/tcr-20-1028). The authors have no conflicts of interest to declare.

Ethical Statement: The authors are accountable for all aspects of the work in ensuring that questions related to the accuracy or integrity of any part of the work are appropriately investigated and resolved. Informed consent was signed by the patients before treatment. The research was in compliance of the Declaration of Helsinki (as revised in 2013). This is a retrospective study on patient data retrieved from hospital medical record system and the patient's personal data have been secured. Ethical approval was obtained from the Institutional Review Board of the Cancer Hospital, Chinese Academy of Medical Sciences (ID: NCC2019C-027).

Open Access Statement: This is an Open Access article distributed in accordance with the Creative Commons Attribution-NonCommercial-NoDerivs 4.0 International
License (CC BY-NC-ND 4.0), which permits the noncommercial replication and distribution of the article with the strict proviso that no changes or edits are made and the original work is properly cited (including links to both the formal publication through the relevant DOI and the license). See: https://creativecommons.org/licenses/by-nc-nd/4.0/.

\section{References}

1. Nascimento de Aquino S, Silvestre Verner F, Alvares Cabral R, et al. Adenoid cystic carcinoma with myoepithelial predominance affecting maxilla and maxillary sinus. J Stomatol Oral Maxillofac Surg 2019;120:55-60.

2. Wolkow N, Jakobiec FA, Lee H, et al. Long-term Outcomes of Globe-Preserving Surgery with Proton Beam Radiation for Adenoid Cystic Carcinoma of the Lacrimal Gland. Am J Ophthalmol 2019;201:84-5.

3. Xu MJ, Wu TJ, van Zante A, et al. Mortality risk after clinical management of recurrent and metastatic adenoid cystic carcinoma. J Otolaryngol Head Neck Surg 2018;47:28.

4. Zhang Y, Liu H, Wu Q, et al. Predictors of Distant Metastasis and Survival in Adenoid Cystic Carcinoma of the External Auditory Canal. Otol Neurotol 2019;40:e1006-11.

5. Scuderi V, Ceriello A, Romano M, et al. Recurrent adenoid cystic carcinoma in the liver: a repeated laparoscopic surgical approach. Updates Surg 2011;63:301-6.

6. Susumu Shingaki, Shohei Kanemaru, Yohei Oda, et al. Distant metastasis and survival of adenoid cystic carcinoma after definitive treatment. J Oral Maxillofac Surg Med Pathol 2014;26:321-16.

7. Seok J, Lee DY, Kim WS, et al. Lung metastasis in adenoid cystic carcinoma of the head and neck. Head Neck 2019;41:3976-83.

8. Zemni I, Tounsi N, Bouraoui I, et al. A Single Liver Metastasis From Adenoid Cystic Carcinoma of the Parotid Gland: Case Report. J Investig Med High Impact Case Rep 2019;7:2324709619879631.

9. Sparchez Z, Mocan T, Hajjar NA, et al. Percutaneous ultrasound guided radiofrequency and microwave ablation in the treatment of hepatic metastases. A monocentric initial experience. Med Ultrason 2019;21:217-24.

10. Eisenhauer EA, Therasse P, Bogaerts J, et al. New response evaluation criteria in solid tumours: Revised RECIST guideline (version 1.1). Eur J Cancer 2009;45:228-47.

11. Shahabi S, Nathan LM, Chanana C, et al. Liver metastasis in a case of adenoid cystic carcinoma of the 
Bartholin's gland: a rare presentation. Arch Gynecol Obstet2009;279:747-50.

12. Harish K, Mangala Gouri SR. Adenoid cystic carcinoma of the parotid metastasizing to liver: case report. BMC Cancer 2004;4:41.

13. Zeidan BA, Abu Hilal M, Al-Gholmy M, et al. Adenoid Cystic Carcinoma of the lacrimal gland metastasising to the liver: report of a case. World J Surg Oncol 2006;4:66.

14. Deshpande AH, Kelkar AA. Hepatic metastasis as an initial manifestation of salivary adenoid cystic carcinoma: Cytologic diagnosis. Diagn Cytopathol 2009;37:45-7.

15. Balducci G, Sagnotta A, Muroni M, et al. An unusual case of exclusive liver metastases from adenoid cystic carcinoma of the submandibular gland: a role for surgery? Report of a case. Surg Today 2011;41:596-9.

16. Akhavan A, Binesh F, Navabii H. Adenoid cystic carcinoma of hard palate with coincidental metastases to lung and liver. BMJ Case Rep 2013;2013:bcr0120125658.

17. Spolverato G, Fite J, Bishop J, et al. Liver metastasis as the initial presentation of adenoid cystic carcinoma. Dig Dis Sci 2014;59:2004-6.

18. Sali PA, Yadav KS, Bushan K, et al. A rare case of lacrimal adenoid cystic carcinoma with large hepatic and multiple pulmonary metastases with successful surgical treatment. Int J Surg Case Rep 2016;20:151-4.

19. Maamari RN, Custer PL, Harocopos GJ. Incidentally Discovered Adenoid Cystic Carcinoma of the Lacrimal Gland with Isolated Liver Metastases. Ocul Oncol Pathol 2017;3:262-6.

20. Hashimoto S, Sumida Y, Tobinaga S, et al. Liver resection for metastases of tracheal adenoid cystic carcinoma: Report of two cases. Int J Surg Case Rep 2018;48:26-9.

Cite this article as: Zheng Y, He Y, Wu F, Liu M, Wang L, $\mathrm{Wu} \mathrm{J}$. Possible local treatment for liver metastases of adenoid cystic carcinoma (ACC): single-centre experience and literature review. Transl Cancer Res 2020;9(8):4573-4582. doi: 10.21037/tcr20-1028
21. Picchia S, Riddell A, Terlizzo M, et al. Liver metastasis from adenoid cystic carcinoma: imaging and histologic features. Curr Probl Cancer 2019;43:331-5.

22. Rapidis AD, Givalos N, Gakiopoulou H, et al. Adenoid cystic carcinoma of the head and neck. Clinicopathological analysis of 23 patients and review of the literature. Oral Oncol 2005;41:328-35.

23. Fordice J, Kershaw C, El-Naggar A, et al. Adenoid cystic carcinoma of the head and neck: predictors of morbidity and mortality. Arch Otolaryngol Head Neck Surg 1999;125:149-52.

24. van der Wal JE, Becking AG, Snow GB, et al. Distant metastases of adenoid cystic carcinoma of the salivary glands and the value of diagnostic examinations during follow-up. Head Neck 2002;24:779-83.

25. Tatari MM, Anajar S, Hassnaoui J, et al. Nasopharyngeal adenoid cystic carcinoma, suggestion of therapeutic innovations: A case report and review of literature. Ann Med Surg (Lond) 2018;30:32-5.

26. Dillon PM, Chakraborty S, Moskaluk CA, et al. Adenoid cystic carcinoma: A review of recent advances, molecular targets, and clinical trials. Head Neck 2016;38:620-7.

27. Wang C, Li T, Yan F, et al. Effect of simvastatin and microRNA-21 inhibitor on metastasis and progression of human salivary adenoid cystic carcinoma. Biomed Pharmacother 2018;105:1054-61.

28. Argiris A, Ghebremichael M, Burtness B, et al. A phase 2 trial of bortezomib followed by the addition of doxorubicin at progression in patients with recurrent or metastatic adenoid cystic carcinoma of the head and neck: a trial of the Eastern Cooperative Oncology Group (E1303). Cancer 2011;117:3374-82. 